\title{
Conditional large Fock state preparation and field state reconstruction in Cavity QED
}

\author{
M. França Santos ${ }^{1}$, E. Solano ${ }^{1,2}$ and R. L. de Matos Filho ${ }^{1}$ \\ ${ }^{1}$ Instituto de Fúsica, Universidade Federal do Rio de Janeiro, Caixa Postal 68528, 21945-970 Rio de Janeiro, RJ, Brazil \\ ${ }^{2}$ Sección Física, Departamento de Ciencias, Pontificia Universidad Católica del Perú, Apartado 1761, Lima, Peru
}

(February 2nd, 2001)

\begin{abstract}
We propose a scheme for producing large Fock states in Cavity QED via the implementation of a highly selective atom-field interaction. It is based on Raman excitation of a three-level atom by a classical field and a quantized field mode. Selectivity appears when one tunes to resonance a specific transition inside a chosen atom-field subspace, while other transitions remain dispersive, as a consequence of the field dependent electronic energy shifts. We show that this scheme can be also employed for reconstructing, in a new and efficient way, the Wigner function of the cavity field state.
\end{abstract}

PACS number(s): 42.50.Dv,32.80.-t,03.65.-w

Harmonic oscillators have been, from the very beginning, at the core of the quantum theory. It was not until the invention of the laser [1], however, that their most interesting statistical properties could be tested in controlled experiments associated with electromagnetic fields. Since then, considerable theoretical and experimental efforts have been devoted to the production and characterization of nonclassical states of light, such as sub-Poissonian [2], squeezed [3], or Schrödinger cat states [4, [5].

A great advance in the field came with the micromaser [6], where two-level Rydberg atoms interact with one mode of a high- $\mathrm{Q}$ cavity in an experimental realization of the Jaynes-Cummings (JC) model [7]. Many important experiments have followed, expliciting the dynamical properties of this model, such as the observation of collapse and revivals [8] and the discrete character of the Rabi oscillations [9] of the atom-field doublets. The micromaser technique has also allowed the investigation of the most fundamental, nonclassical states of harmonic oscillators, specially the number (Fock) states. In particular, recent papers reported the QND measurement of the one photon Fock state [10] and the preparation of up to two photons [11] in the cavity mode.

The atom-field dynamics as well as the statistical properties of the field are observed, in Cavity QED, through the detection of the atoms, which work either as a probe for the coupled system or as a measuring device for the light mode state, depending on the setup. Indeed, several theoretical results have showed that appropriate settings of this system enable a complete reconstruction of the quantum state of the cavity field [12,13]. However, despite the great advances in the latest years, the implementation of these proposals, as well as the generation and characterization of large Fock states remain as challenging experimental problems in this field. In these cases, the characteristics of the JC model require the use of improved apparatus, such as cavities with higher quality factor and enhanced ways to control and manipulate the atoms.

Alternatively, different atom-field couplings could be used. In particular, selective interactions 14 present new ways to induce transitions in this system. Unlike the JC model, where the same resonant or dispersive regime applies for all initial atom-field states, selective interactions separate these states in subspaces with distinct coupling regimes. This property represents more versatility and the possibility to implement new classes of experiments even under current technological conditions.

In this paper, we show that it is possible to implement a selective interaction between three level atoms, a classical field and a quantized cavity mode. We study the feasibility of our scheme based on available experimental parameters and propose, as a first application, the preparation of large Fock states in the quantized mode. As a second relevant application, we show that selectivity in this system allows for the measurement of the probability distribution $P(n)$ of the quantized field state in the Fock state basis $|n\rangle$. Finally, we show that it is possible, as a natural consequence, to propose an efficient reconstruction method of the Wigner function 15 of the cavity field and, therefore, of its complete quantum state [16].

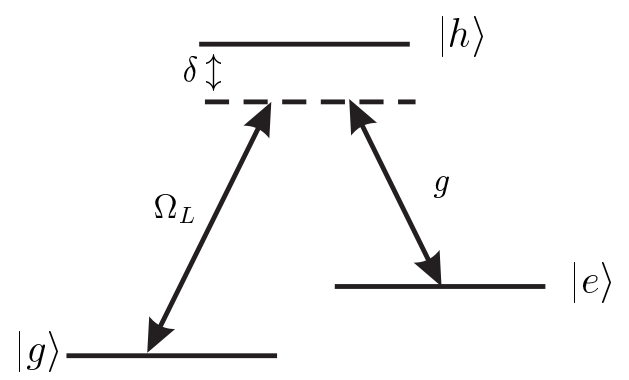

FIG. 1. Scheme for the Raman excitation of the three-level atom.

Our proposal relies on the Raman excitation of a three level atom by a classical field of frequency $\omega_{L}$ and a quantized cavity mode of frequency $\omega_{0}$, in a lambda configuration (see Fig. 1). The classical field drives dispersively 
the transition from level $|g\rangle$ to level $|h\rangle$, with coupling constant $\Omega_{L}$ and detuning $\delta=\omega_{h g}-\omega_{L} \gg\left|\Omega_{L}\right|$. The cavity mode couples level $|e\rangle$ to level $|h\rangle$, with coupling constant $g$ and the same detuning $\delta=\omega_{h e}-\omega_{0} \gg|g|$. In the interaction picture, the interaction Hamiltonian in the RWA approximation is given by

$$
\widehat{H}_{\text {int }}=\hbar \Omega_{L} \widehat{\sigma}_{h g} e^{-i \delta t}+\hbar g \widehat{\sigma}_{h e} \widehat{a} e^{-i \delta t}+\text { H.c. },
$$

where $\widehat{\sigma}_{j m} \equiv|j\rangle\langle m|$ is an electronic flip operator and $\hat{a}$ is the annihilation operator of the quantized cavity mode. Since level $|h\rangle$ is coupled dispersively with both levels $|g\rangle$ and $|e\rangle$, it can be adiabatically eliminated giving rise to an effective second order anti-Jaynes-Cummings Hamiltonian

$$
\begin{aligned}
\widehat{H}_{\mathrm{eff}}= & \hbar \frac{\left|\Omega_{L}\right|^{2}}{\delta} \widehat{\sigma}_{g g}+\hbar \frac{|g|^{2} \widehat{a}^{\dagger} \widehat{a}}{\delta} \widehat{\sigma}_{e e} \\
& +\hbar \frac{\left|g \Omega_{L}^{*}\right|}{\delta}\left(\widehat{\sigma}_{e g} \widehat{a}^{\dagger}+\widehat{\sigma}_{g e} \widehat{a}\right),
\end{aligned}
$$

where we chose $\Omega_{L}$ in phase with $g$. The first two terms correspond to dynamical energy shifts of levels $|g\rangle$ and $|e\rangle$, and the last two terms describe transitions between these levels, accompanied by creation or annihilation of a photon in the cavity mode. Notice that the difference of the energy shifts of level $|g\rangle$ and $|e\rangle$, which depends explicitly on the number $n$ of photons in the cavity mode, will determine the effective resonance frequency of the $|g\rangle \leftrightarrow|e\rangle$ transition.

The Hamiltonian (2) is block separable in the subspaces spanned by the states $\{|g, n\rangle,|e, n+1\rangle\}$ of the atom-field system. There is a specific difference of energy shifts $\Delta_{e g}^{n}$, associated to each one of these subspaces, which may be compensated by external action on either the atom (dc Stark shift) or the cavity mode (by shifting its frequency). In this way, transitions inside a chosen subspace may be tuned to resonance, while other transitions remain dispersive, producing a selective interaction in the atom-field Hilbert space. Once this frequency adjustment is made for one specific subspace $\left\{\left|g, N_{o}\right\rangle,\left|e, N_{o}+1\right\rangle\right\}$, the detunings associated with the remaining subspaces $\left(n \neq N_{o}\right)$ change to

$$
\Delta_{n} \equiv \Delta_{e g}^{n}-\Delta_{e g}^{N_{o}}=\frac{|g|^{2}}{\delta}\left(n-N_{o}\right) .
$$

By controlling the ratio between $g$ and $\Omega_{L}$, the detunings $\Delta_{n}$ can be made large enough for considering the effective interaction in the remaining subspaces as dispersive, i.e., $\Delta_{n} \gg \frac{\left|g \Omega_{L}^{*}\right|}{\delta}$. In this case, if the atom enters the cavity in state $|g\rangle$, it can only experiment a Rabi flip to level $|e\rangle$ if the cavity has $N_{o}$ photons.

Hamiltonian (2) can be easily diagonalized. In particular, after the frequency adjustment made in Eq. (3), its stationary eigenstates are given by the ground state $|e, 0\rangle$ and the doublets

$$
| \pm, n\rangle=\frac{G_{n}|g, n\rangle+\lambda_{ \pm, n}|e, n+1\rangle}{\sqrt{\lambda_{ \pm, n}^{2}+G_{n}^{2}}},
$$

with respective eigenvalues 0 and $\lambda_{ \pm, n}=\frac{\Delta_{n}}{2} \pm \Omega_{n}$. In Eq. (㓙), $G_{n}=\frac{\left|g \Omega_{L}^{*}\right|}{\delta} \sqrt{n+1}$ and $\Omega_{n}=\sqrt{\Delta_{n}^{2} / 4+G_{n}^{2}}$.

For arbitrary values of the detunings $\Delta_{n}$, if the atom enters the cavity in state $|g\rangle$, and the field is initially in state $\left|\Phi_{0}\right\rangle=\sum_{n} c_{n}|n\rangle$, the state of the system evolves, after an interaction time $t$, to

$$
\begin{aligned}
|\Psi(t)\rangle= & \sum_{n} c_{n} e^{-i \frac{\Delta_{n} t}{2}}\left[\left(\cos \Omega_{n} t+\frac{i \Delta_{n}}{2 \Omega_{n}} \sin \Omega_{n} t\right)|g, n\rangle\right. \\
& \left.-\frac{i G_{n}}{\Omega_{n}} \sin \Omega_{n} t|e, n+1\rangle\right] .
\end{aligned}
$$

In particular, if the atom is found in state $|e\rangle$, after it has interacted with the light fields during a time interval $\tau=\frac{\pi \delta}{2\left|g \Omega_{L}^{*}\right| \sqrt{N_{o}+1}}$, the correlated state of the cavity mode is

$$
\left|\Phi_{e}(\tau)\right\rangle=\frac{c_{N_{o}}\left|N_{o}+1\right\rangle+\sum_{n \neq N_{o}} b_{n}|n\rangle}{\sqrt{\left|c_{N_{o}}\right|^{2}+\sum_{n \neq N_{o}}\left|b_{n}\right|^{2}}} .
$$

The coefficients $b_{n}$ are given by

$$
b_{n}=\frac{c_{n}(-i) e^{-i \frac{\Delta_{n} \tau}{2}} \sin \frac{\pi}{2} \sqrt{\frac{q}{N_{o}+1}}}{\sqrt{q}}
$$

where $q=\frac{r^{2}\left(n-N_{o}\right)^{2}}{4(n+1)}+1$, and $r=\frac{|g|}{\left|\Omega_{L}\right|}$. Note that, as $q$ increases, the coefficients $b_{n}$ become negligible compared to the coefficient $c_{N_{o}}$ and $\left|\Phi_{e}(\tau)\right\rangle$ tends to the Fock state $\left|N_{o}+1\right\rangle$. For a chosen $N_{o}$, this condition is satisfied if $r \gg 2 \sqrt{N_{o}+2}$. In this limit, the Fock state $\left|N_{o}+1\right\rangle$ could be produced in the cavity field, as long as $c_{N_{o}} \neq 0$. In principle, one could use this scheme to produce any Fock state in the quantized mode. In practice, however, one is limited by the decay time of the cavity, $\tau_{c}$, which must be much longer than the interaction time, $\tau$. Typically, for Rydberg atoms interacting with high-Q microwave cavities, $g / 2 \pi \sim 50 \mathrm{kHz}$ [10]. For $N_{o} \sim 10, \delta / 2 \pi \sim 1 \mathrm{MHz}$ and $\Omega_{L} \sim \frac{g}{30}(r \sim 30), \tau$ will be of the order of $1 \mathrm{~ms}$. Interaction times of this order are still much shorter than the decay time of the microwave cavities with highest quality factor used nowadays (of the order of $0.3 \mathrm{~s}$ [17]). This suggests that, in principle, our proposal could be implemented immediately.

We may define the fidelity of generating the selected Fock state $\left|N_{o}+1\right\rangle$ as $F \equiv\left|\left\langle N_{o}+1 \mid \Phi_{e}(\tau)\right\rangle\right|^{2} . F$ is approximately given by $1-\sum_{n} \frac{\left|b_{n}\right|^{2}}{\left|c_{N_{o}}\right|^{2}}$ and it approaches unity when the coefficients $b_{n}$ 's go to zero. Initial states for which $c_{N_{o}}>c_{n}$ enhance the protocol efficiency. In this sense, good candidates for initial cavity state are coherent states with mean number of photons around $N_{0}$. Not only do they satisfy $c_{N_{o}}>c_{n}$, but they are also easily produced in microwave cavities, by just coupling them to a microwave generator [5]. In Fig. 2, we show an example for the preparation of large Fock states in the cavity mode, after only one atom has interacted with the fields. 
From an initial coherent state $|\alpha\rangle$ with $|\alpha|^{2}=5$, the Fock state $|6\rangle$ is prepared in the cavity with a fidelity higher than 0.99 .
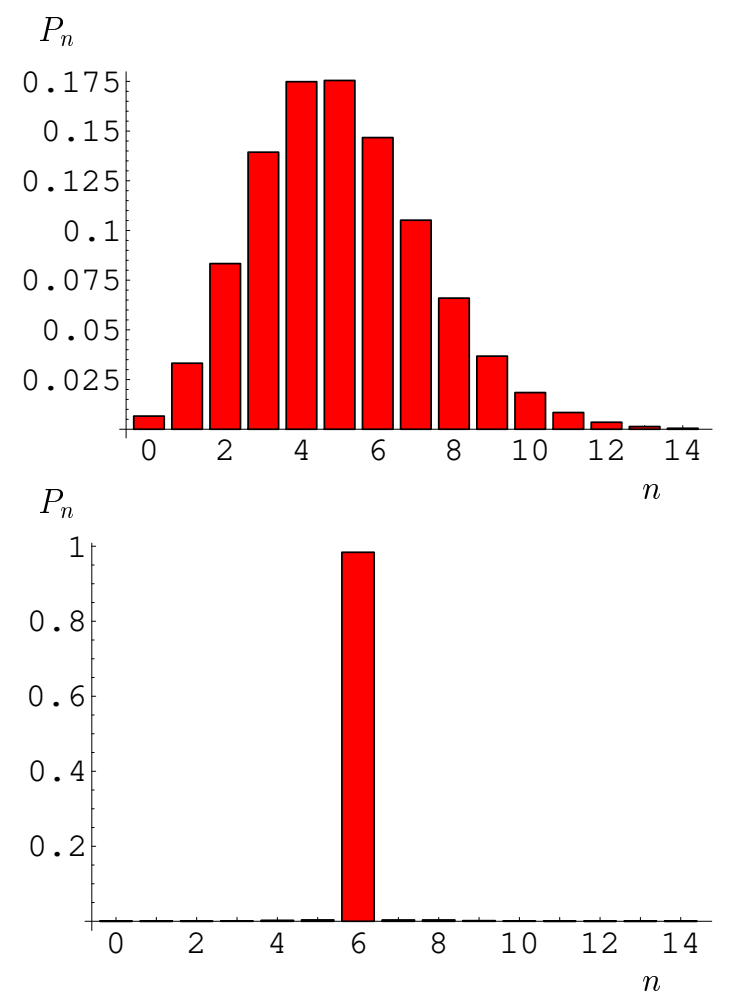

FIG. 2. Preparation of the Fock state $|6\rangle$ in the cavity mode by measuring the atom in its excited state after it passed through the cavity. The cavity field was initially in a coherent state with $|\alpha|^{2}=5$. The value of the parameter $r$ was set to $r=30$.

The Fock state preparation is conditioned to finding the atom in state $|e\rangle$. From Eqs. (5), (6) and (7), it is easy to show that as $r$ becomes larger, the probability $P_{e}$ of measuring the atom in the excited state approximates the probability $P_{N_{o}}$ of finding $N_{o}$ photons in the initial cavity field state. If $r$ cannot be made large enough due to experimental limitations, the state one wants to prepare is polluted by marginal Fock states populations. In this case, one only needs to send a second atom in state $|g\rangle$ and set the experimental parameters to the transition $\left|g, N_{o}+1\right\rangle \rightarrow\left|e, N_{o}+2\right\rangle$. The probability of finding both atoms in the excited state becomes closer to $P_{N_{o}}$, and the field state produced will be, with very high fidelity, state $\left|N_{o}+2\right\rangle$.

The equivalence between $P_{e}$ and $P_{N_{o}}$ for large $r$ suggests a very practical and easy way to obtain the photon statistics $P_{n}$ of an arbitrary state in the cavity mode. In fact, for each selected transition $|g, N\rangle \leftrightarrow|e, N+1\rangle$, the proportion of atoms measured in state $|e\rangle, P_{e}$, gives directly $P_{N}$, for all possible values of $N$. Combined with the possibility of coherently displacing microwave cavity fields, this allows one to fully reconstruct the Wigner function of the state of the quantized mode. Since it does not rely on additional devices, such as Ramsey interferometers, this scheme simplifies the task of field state reconstruction, as we will discuss below.

The Wigner function of the state $\hat{\rho}$ of a harmonic oscillator, can be written as

$$
W(-\alpha)=\frac{2}{\pi} \sum_{n}(-1)^{n} P_{n}(\alpha),
$$

where $P_{n}(\alpha)=\left\langle n\left|\widehat{D}(\alpha) \hat{\rho} \widehat{D}^{-1}(\alpha)\right| n\right\rangle$ is the number distribution of state $\hat{\rho}$ displaced coherently in the phase space by $\alpha$ [16.18]. This tells us that, to obtain the Wigner function of the cavity field on each point of the phase space, all one needs to know is the number distribution of the field, after it has been displaced in the phase space. The coherent displacement of the cavity field can be easily implemented by coupling the cavity to a microwave generator. After this step, one can use the selective scheme discussed above to measure $P_{n}(\alpha)$ and, then, Eq. (8) to calculate $W(-\alpha)$. This method is exact for large values of $r$, and it represents an experimentally simple way to measure the photonic statistics of the cavity field and to reconstruct its Wigner function. For once, it does not require the preparation of atoms in a coherent superposition of upper and lower states, as in usual schemes that rely on Ramsey interferometry. This simplifies the experimental setup and, more important, permits the use of closed higher quality cavities with characteristic lifetimes much longer than the interaction times in question. As an example, we show in Fig. 3 the efficient reconstruction of the Wigner function of the Fock state $|6\rangle$ for the realistic parameter $r=30$. By subtracting the exact Wigner function from the reconstructed one, we also show that the errors introduced by suposing that transitions occur only inside each selected subspace $|g, N\rangle \leftrightarrow|e, N+1\rangle$ (perfect selectivity) are negligibly small.

In conclusion, we have proposed a scheme to implement a selective interaction in cavity QED, between three level atoms, a classical field and a quantized cavity mode. It relies on the possibility of tuning to resonance a specific transition inside a chosen atom-field subspace, while other transitions remain dispersive, as a consequence of the field dependent electronic energy shifts. As a first relevant application of this scheme, we have proposed a method for generating large Fock states in the cavity mode. Aditionally, we have shown that this scheme allows for the reconstruction of the Wigner function of an arbitrary cavity field state, with simplified experimental setup. Many other application examples could be imagined, specially those exploring the entanglement created between the atoms and different field states, as is the case in quantum logic and quantum communication schemes.

We want to thank the hospitality of the organizers of the Pan-American Advanced Study Insitute on "Chaos, Decoherence and Quantum Entanglement" and S. Haroche for fruitful discussions about the experimental implementation of the proposed scheme. 
This work has been supported by Conselho Nacional de Desenvolvimento Científico e Tecnológico (CNPq), Fundação de Amparo à Pesquisa do Estado do Rio de Janeiro (FAPERJ), Fundação Universitária José Bonifácio (FUJB), and Programa de Apoio a Núcleos de Excelência (PRONEX).
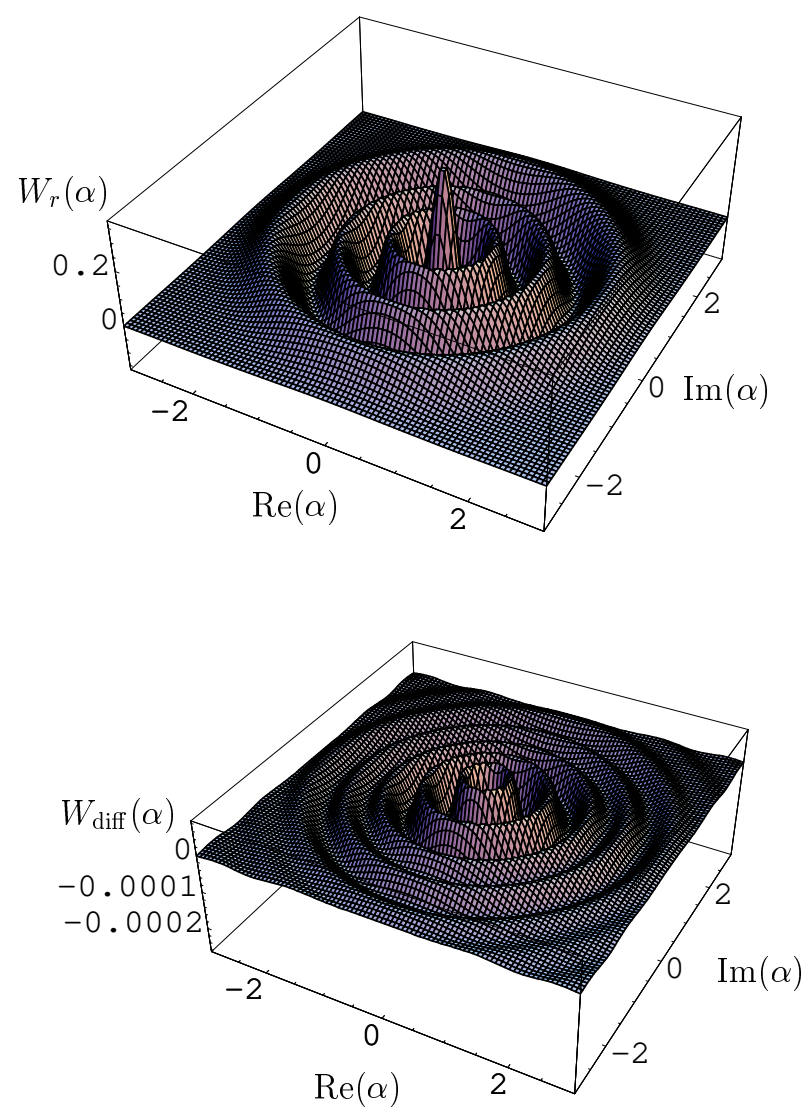

FIG. 3. Plot of the reconstructed Wigner function of the Fock state $|6\rangle$ with the use of the selective scheme (above) and its difference to the exact one (below). The value of the parameter $r$ was set to $r=30$.

[1] F. T. Arecchi, Phys. Rev. Lett. 15, 912 (1965); M. O. Scully and W. E. Lamb, Jr., Phys. Rev. 159, 208 (1967); H. Haken in Handbuch der Physik, edited by L. Genzel (Springer, Berlin, 1970), Vol. XXV/2C .

[2] H. J. Kimble, M. Dagenais, and L. Mandel, Phys. Rev. Lett. 39, 691 (1977); Phys. Rev. A 18, 201 (1978); R. Short and L. Mandel, Phys. Rev. Lett. 51, 384 (1983).

[3] R.E. Slusher et al., Phys. Rev. Lett. 55, 2409 (1985); J. Opt. Soc. Am. B 41449 (1987), special issue on squeezed states of the electromagnetic field, edited by H. J. Kim- ble and D. F. Walls; J. Mod. Opt. 34707 (1987), special issue on squeezed light, edited by R. Loudon and P. L. Knight; Appl. Phys. B: Photophys. Laser Chem. 55189 (1992), special issue on quantum noise reduction in optical systems-experiments, edited by E. Giacobino and C. Fabre.

[4] E. Schrödinger, Naturwissenschaften 23, 807(1935).

[5] L. Davidovich, M. Brune, J.M. Raimond, and S. Haroche, Phys. Rev. A 53, 1295 (1996); M. Brune, E. Hagley, J. Dreyer, X. Maitre, A. Maali, C. Wunderlich, J.M. Raimond, and S. Haroche, Phys. Rev. Lett. 77, 4887 (1996).

[6] For a review on micromaser, see Marlan O. Scully and M. Suhail Zubairy Quantum Optics (Cambridge University Press, Cambridge, 1997).

[7] E.T. Jaynes and F.W. Cummings, Proc. IEEE 51, 89 (1963).

[8] G. Rempe and H. Walther, Phys. Rev. Lett. 58353 (1987).

[9] M. Brune, F. Schmidt-Kaler, A. Maali, J. Dreyer, E. Hagley, J. M. Raimond, and S. Haroche, Phys. Rev. Lett. 76, 1800 (1996).

[10] G. Nogues, A. Rauschenbeutel, S. Osnaghi, M. Brune, J. M. Raimond and S. Haroche, Nature 400, 239 (1999).

[11] B.T.H. Varcoe, S. Brattke, M. Weidinger, and H. Walther, Nature 403, 743 (2000)

[12] M. Wilkens and P. Meystre, Phys. Rev. A 43, 3832 (1991); S.M. Dutra, P.L. Knight and H. Moya-Cessa, Phys. Rev. A 48, 3168 (1993); S.M. Dutra and P. L. Knight, Phys. Rev. A 49, 1506 (1994); C. T. Bodendorf, G. Antesberger, M. S. Kim, and H. Walther, Phys. Rev. A 57, 1371 (1998); M. S. Kim, G. Antesberger, C.T. Bodendorf, and H. Walther, Phys. Rev. A 58 , R65 (1998).

[13] L. G. Lutterbach and L. Davidovich, Phys. Rev. Lett. 78, 2547 (1997).

[14] T. Pellizzari and H. Ritsch, Phys. Rev. Lett. 72, 3973 (1994); E. Solano, P. Milman, R. L. de Matos Filho, and N. Zagury, Phys. Rev. A 62, 021401(R) (2000); H. Nha, Y.-T. Chough, and K. An, Phys. Rev. A, 63, 010301 R (2001). See also Ref. 10.

[15] E. Wigner, Phys. Rev. 40, 749 (1932); for a review, see M. Hillery, R. F. O'Connel, M.O. Scully, and E. P. Wigner, Phys. Rep. 106, 121 (1984).

[16] K.E. Cahill and R. J. Glauber, Phys. Rev. 177, 1857 (1969); ibid 177, 1882 (1969);

[17] B.T.H. Varcoe, S. Brattke, and H. Walther, J. Opt. B, 2, 154 (2000).

[18] K. Banaszek and K. Wódkiewicz, Phys. Rev. Lett. 76, 4344 (1996); S. Wallentowitz and W. Vogel, Phys. Rev. A 53, 4528 (1996). 